$\mathbb{T}$ periodica polytechnica

\author{
Transportation Engineering \\ $36 / 1-2(2008) 99-104$ \\ doi: 10.3311/pp.tr.2008-1-2.18 \\ web: http://www.pp.bme.hu/tr \\ (c) Periodica Polytechnica 2008
}

RESEARCH ARTICLE

\section{The design of a brake control to improve road holding}

\author{
Gábor Rödönyi / Péter Gáspár
}

Received 2007-03-03

\begin{abstract}
A robust control synthesis method is presented for an emergency steer-by-brake problem of a heavy truck. The goal is to provide automated steering function for a truck with a mechanic-pneumatic steering system, where the only possibility for automated intervention is the use of the electronic brake system. This problem is motivated by situations when the driver becomes incapable of controlling the vehicle due to some lipothymy or drowsiness. Assuming an emergency detection system and a higher level control strategy that defines yaw-rate reference for navigating the vehicle, the low level reference tracking control is designed by the $\mathcal{H}_{\infty}$ method and illustrated by Matlab/Simulink simulation.
\end{abstract}

\section{Keywords}

robust control $\cdot$ uncertainty modeling $\cdot$ steer-by-brake $\cdot$ path following

\section{Acknowledgement}

This work was supported by the Hungarian National Science Foundation (OTKA) under the grant $T-048482$ which are gratefully acknowledged.

\section{Gábor Rödönyi}

Computer and Automation Research Institute, Hungarian Academy of Sciences, Kende u. 13-17, H-1111 Budapest,, Hungary

\section{Péter Gáspár}

Computer and Automation Research Institute, Hungarian Academy of Sciences, Kende u. 13-17, H-1111 Budapest,, Hungary

e-mail: gaspar@sztaki.hu

\section{Introduction}

The aim of preventing rollovers is to provide the vehicle with an ability to resist overturning moments generated during cornering. Several schemes with possible active intervention into the vehicle dynamics have been proposed. One of these methods employs active anti-roll bars, that is, a pair of hydraulic actuators which generate a stabilizing moment to balance the overturning moment, see [1, 9, 12]. Another method applies active steering since it affects lateral acceleration directly, see [3], [2]. The third method applies an electronic brake mechanism to reduce the lateral tire forces acting on the outside wheel, see [5, 6, 10].

In this paper a complex control structure is presented in order to reduce the lateral acceleration of the vehicle. The controller directly desires brake pressures of the wheels on the one side. The vehicle model consists of four components in serial connection as follows. A single-track model (denoted by $B$ in Fig. 1) for describing the yaw dynamics; the nonlinear wheel dynamics $W$; the brake actuator $A$; and the static, nonlinear tire-road contact force model $C$. The structure of the controller corresponds to structure of the model. The controller $K_{B}$ is designed based on the yaw model $B$ in order to reduce the lateral acceleration. The contact force model inversion $C^{-1}$, the slip controller $K_{W}$ and the actuator controller $K_{A}$ are to realize the brake forces desired by the controller $K_{B}$.

The controllers are switched on only for a short period. During this period the velocity of the vehicle does not change significantly. Therefore, after linearization, all the three controllers are linear time-invariant controllers that are designed to be robust against worst case disturbances and modelling errors.

The paper is organized as follows: Section 2 presents the vehicle model, the base of the controller synthesis. In section 3 the control design procedure is detailed. Section 4 is to demonstrate the efficiency of the controller on an overtaking manoeuvre.

\section{The vehicle model for control}

The goal of the design is to reduce rollover risk by decreasing lateral acceleration $a_{y}$ of the vehicle beyond a critical level. The concept is to switch on a controller when $a_{y}$ is above this level. The braking of the outer side of the vehicle generates a yaw 
moment and decreases the lateral tire forces. The drawback is that the side slip of the vehicle will increase.

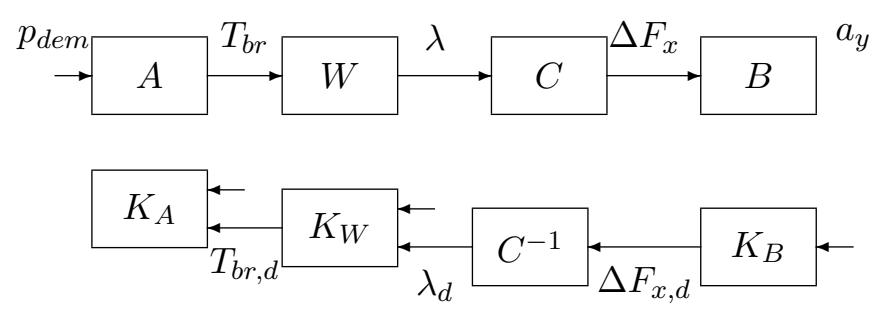

Fig. 1. Cascade control scheme

This approach does not require any measurements of the state of the sprung mass like for example roll rate. It is enough to model the yaw dynamics and tire-characteristics and to use the common EBS measurements, i.e. lateral and longitudinal acceleration, yaw rate $r$, brake pressures $p$ and wheel velocities $v_{R}$.

The equations of motion for control design is derived from a 17-degree-of-freedom nonlinear model of a MAN truck that contains the dynamics of suspension, yaw, roll, pitch, heave motions, steering systems, wheel and brake actuator dynamics [11]. The model for control is written as a single track model plus the nonlinear effect of the contact forces. Assuming the change of contact forces is well approximated, the resulted model attains good fit to the nonlinear one and might be valid as long as all the four wheels contact the ground. The equations of motion of the single-track model $B$ are described as

$$
\begin{aligned}
\dot{x}_{B} & =A x_{B}+B_{\delta} \delta+B_{x} \Delta F_{x}+B_{y} \Delta F_{y} \\
\dot{x}_{w, i j} & =f_{w}+g_{w} T_{b r, i j} \\
\dot{x}_{A, i j} & =A_{b r} x_{A, i j}+B_{b r} p_{d e m, i j} \\
T_{b r, i j} & =C_{b r} x_{A, i j}+D_{b r} p_{d e m, i j}
\end{aligned}
$$

$$
\begin{gathered}
\text { where } A=\left[\begin{array}{cc}
-\frac{c_{f}+c_{r}}{v m} & -1+\frac{c_{r} l_{r}-c_{f} l_{f}}{v^{2} m} \\
\frac{c_{r} l_{r}-c_{f} l_{f}}{J_{z}} & -\frac{c_{r} l_{r}^{2}+c_{f} l_{f}^{2}}{v J_{z}}
\end{array}\right], \\
B_{\delta}=\left[\begin{array}{c}
\frac{c_{f}}{v m} \\
\frac{c_{f} l_{f}}{J_{z}}
\end{array}\right], B_{x}=\left[\begin{array}{c}
0 \\
\frac{l_{w}}{2 J_{z}}
\end{array}\right], B_{y}=\left[\begin{array}{cc}
\frac{1}{v m} & \frac{1}{v m} \\
\frac{l_{f}}{J_{z}} & \frac{-l_{r}}{J_{z}}
\end{array}\right], \\
f_{w}=-F_{x, i j} \frac{r_{e f f}}{J_{w} v_{w, i j}}, g_{w}=-\frac{r_{e f f}}{J_{w} v_{w, i j}}, \\
\Delta F_{x}=F_{x f r}-F_{x f l}+F_{y r r}-F_{y r l}, \\
\Delta F_{y}=\left[\begin{array}{c}
\Delta F_{y f} \\
\Delta F_{y r}
\end{array}\right]
\end{gathered}
$$

and the state vector of the yaw dynamics is

$$
x_{B}=[\beta r]^{T} \text {. }
$$

The $\beta, r, \delta$ denote the vehicle slip angle, yaw rate and steering angle, respectively. The state of the wheel dynamics is defined by $x_{w, i j}=\lambda_{i j}-1$, where $\lambda_{i j}=\frac{v_{R, i j}}{v_{w, i j}}$. The tire-road contact point velocity is denoted by $v_{w, i j}$. The indexes $i j$ stand for the four wheels, $f r, f l, r r$ and $r l$. The $A_{b r}, B_{b r}, C_{b r}$ and $D_{b r}$ are parameters of the black-box actuator model. The control signals are the brake pressure demand $p_{d e m, i j}$. The $\Delta F_{y f}$ 's and $\Delta F_{y r}$ are the remaining lateral wheel force $F_{y}$ components after the linearization

$$
\begin{aligned}
& F_{y f}=F_{y f l}+F_{y f r}=c_{f} \alpha_{f}+\Delta F_{y f} \\
& F_{y r}=F_{y r l}+F_{y r r}=c_{r} \alpha_{r}+\Delta F_{y r}
\end{aligned}
$$

The parameters $c_{f}, c_{r}, l_{f}, l_{r}, l_{w}, J_{z}, v, m, r_{e f f}$ and $J_{w}$ denote the front and rear cornering stiffness constants, the front and rear distances between the center of gravity and axles, the length of an axle, inertia of the vehicle around the vertical axis, forward velocity, total mass, effective wheel radius and inertia of the wheel, respectively. The front and rear wheel slip angles are approximated by $\alpha_{f}=\delta-\beta-\frac{l_{f} r}{v}$ and $\alpha_{r}=-\beta+\frac{l_{r} r}{v}$, respectively.

For the tire adhesion model the Burckhardt formula is applied [4] 8]. The longitudinal tire-road contact forces are expressed by the slip $\lambda$ and side slip angle $\alpha$ as follows:

$$
F_{x}=F_{z} C(\lambda, \alpha)(\lambda-\cos (\alpha))
$$

where $C(\lambda, \alpha)=\frac{\mu(s)}{s} \frac{1}{\max (1, \cos (\alpha) \lambda)}, \mu(s)=c_{1}(1-$ $\left.e^{-c_{2} s}\right)-c_{3} s, s=\frac{\sqrt{1+\lambda^{2}-2 \lambda \cos (\alpha)}}{\max (1, \cos (\alpha) \lambda)}$. The vertical wheel load is denoted by $F_{z}$. The function $C(\lambda, \alpha)$ is the cornering stiffness function. It is assumed in this paper that $C(\lambda, \alpha)$ is approximately known by estimating the parameters $c_{1}, c_{2}$ and $c_{3}$, see [8]. It is assumed that the velocity $v$, wheel slip angles $\alpha_{i}$ and the velocity of the tire-road contact point $v_{w, i j}$ are estimated [7. 8].

\section{The design of a robust cascade control}

As described in (1)-3) the vehicle model has three dynamic components in hierarchical structure. Because of the nonlinearity of the wheel model in (2) the control is similarly structured as shown in Fig. 1. The idea of linearization of the wheel model around some operation points was rejected because of the cornering stiffness function's sensitivity of the ever-changing side slip angle.

The controller $K_{B}$ is to reduce the lateral acceleration by manipulating the yaw moment through $\Delta F_{x}$. When $\Delta F_{x}>0$, the left side is braked and $F_{x f r}=F_{x r r}=0$ and consistently when $\Delta F_{x}$ is negative. The model for control synthesis is the singletrack model (1). The steering angle $\delta$ and the term $\Delta F_{y}$ are considered as disturbances in this control problem. The generalized plant for the $\mathcal{H}_{\infty}$ control design is described by the following equations:

$$
\begin{aligned}
\dot{x}_{B} & =A x_{B}+W_{w} w+B_{x} \Delta F_{x} \\
z_{1} & =W_{z 1} C_{1} x_{B} \\
z_{2} & =W_{z 2} \Delta F_{x}
\end{aligned}
$$


turbance vector, $W_{w}, W_{z 1}, W_{z 2}$ and $W_{n}$ denote the frequency 
weighting filters for the disturbance $w$, the two performance signals $z_{1}$ and $z_{2}$ and the sensor noise $n$, respectively. The performance output $z_{1}$ penalizes the lateral acceleration and is derived from $a_{y}=v(\dot{\beta}+r)$ with the disturbance terms excluded.

When choosing $W_{w}$ and implementing the controller it should be considered that the steering angle, which is a disturbance here, has much larger effect on the vehicle than the control input $\Delta F_{x}$. The braking should serve only as a slight modifier of the yaw dynamics and must not work in normal driving situations. The traditional solution in similar cases used to be the design of the controller without considering the large and measurable disturbance term, then the controller is implemented by feeding it by the plant output subtracted by the output of a reference model driven by the disturbance. Finally one has to care about proper reference signal. Instead of this computationally effortful feedforward technics a very simple method is applied. The lateral acceleration is driven through a dead-zone nonlinearity to the controller, i.e. the controller is fed by a nonzero input only if the lateral acceleration exceeds a certain level, particularly

$$
y=\left\{\begin{array}{l}
\operatorname{sign}\left(a_{y}\right)\left(\left|a_{y}\right|-4 \mathrm{~m} / \mathrm{s}^{2}\right) \text { if }\left|a_{y}\right|>4 \mathrm{~m} / \mathrm{s}^{2} \\
0 \text { otherwise. }
\end{array}\right.
$$

Furthermore, whenever $y=0$ holds the states of the controller are set to zero. Thus, the controller considers only the particle of disturbances that are responsible for lateral acceleration larger than $4 \mathrm{~m} / \mathrm{s}^{2}$. Concerning the disturbance $\Delta F_{y}$, it may be minimized by choosing the cornering stiffness constants $c_{f}$ and $c_{r}$ as the mean values of $F_{z f r} C\left(\lambda_{f r}, \alpha_{f}\right)+F_{z f l} C\left(\lambda_{f l}, \alpha_{f}\right)$ in the range of operation.

The next task is the computation of the slip references for the slip controllers $K_{W}$ of each braked wheels. The following computations are presented in the case of braking the left side, i.e. $\Delta F_{x}=-F_{x f l}-F_{y r l}>0$, the other case trivially follows. The same slip values are prescribed for both braked wheels.

First, the control input of $K_{B}$ is saturated by

$$
\Delta F_{x, \max }=-\kappa \max _{\lambda}\left(F_{z f l} C\left(\lambda, \alpha_{f}\right)\left(\lambda-\cos \left(\alpha_{f}\right)\right)\right)
$$

where $0<\kappa \leq 1$ (say 0.8 ) is to safely avoid the unstable slip region beyond the peak of the friction coefficient function. Second, the monotone part, near the origin, of the function

$$
\lambda \longmapsto-F_{z f l} C\left(\lambda, \alpha_{f}\right)\left(\lambda-\cos \left(\alpha_{f}\right)\right)-F_{z r l} C\left(\lambda, \alpha_{r}\right)\left(\lambda-\cos \left(\alpha_{r}\right)\right)
$$

is inverted at the saturated control input in order to get the slip demand $\lambda_{d}$. The wheel slip controller $K_{W}$ has to drive the system (2) on the trajectory $\lambda_{d}-1$. The output, $T_{b r}$, of the controller is the reference signal for the low level actuator controller $K_{A}$.

The tracking problem of the nonlinear system (2) is solved by feedback linearization and $\mathcal{H}_{\infty}$ control for the linearized plant. For this, $f_{w}$ is rewritten in the form of $f_{w}=A_{w} x_{w}+f$, where the negative scalar $A_{w}$ is chosen so that the $\mathcal{H}_{\infty}$ controller will have numerically tractable and implementable dynamics. The equations of the controller are the following:

$$
T_{b r, d}=\frac{-f+u_{w}}{g_{w}}
$$

where $u_{w}$ is the output of the $\mathcal{H}_{\infty}$ controller designed for the following generalized plant:

$$
\begin{aligned}
\dot{x}_{w} & =A_{w} x_{w}+W_{f} w_{f}+g_{w} u_{w} \\
z_{t} & =W_{z t}\left(W_{d} x_{w, d}-x_{w}\right) \\
z_{u} & =W_{z u} u_{w}
\end{aligned}
$$

where $w_{f}$ denotes the disturbance of the imprecise feedbacklinearization, $z_{t}$ and $z_{u}$ are the performance signals for the tracking and control energy, respectively. The $n_{w}$ is the measurement noise on the slip $x_{w}$. The reference signal is $x_{w, d}=\lambda_{d}-1$. $W_{f}, W_{z t}, W_{z u}, W_{d}$ and $W_{n w}$ denote the appropriate frequency weighting filters.

The goal of the third controller $K_{A}$ is to implement the desired brake torque on the brake actuator, which is the electricpneumatic system between the electronic control unit and brake cylinder. Disturbances are assumed to act only on the output of the actuator, because the actuator dynamics has minor uncertainty, but the brake-torque $T_{b r}$ is computed from the cylinder pressure multiplied by the uncertain brake transmission factor. The generalized plant for the $\mathcal{H}_{\infty}$ controller is the following:

$$
\begin{aligned}
\dot{x}_{A} & =A_{b r} x_{A}+B_{b r} p_{d e m} \\
z_{T} & =W_{z T}\left(W_{T} T_{b r, d}-C_{b r} x_{A}\right) \\
z_{p} & =W_{z p} p_{d e m}
\end{aligned}
$$

where $n_{T}$ denotes the output disturbance, $z_{T}$ and $z_{p}$ are the performance signals for the tracking and control energy, respectively. $W_{z T}, W_{z p}, W_{T}$ and $W_{n T}$ denote the appropriate frequency weighting filters.

\section{Analysis on a test maneuver}

The proposed control method is validated by Matlab simulation on a complete nonlinear vehicle model. The model is a two-track model with four independent suspension systems that describe the heave motion of the wheels. Roll and pitch springdamper systems model the roll and pitch dynamics, respectively, of the chassis. The brake actuators and the dynamics of the steering system are also modelled. The wheel dynamics is the same as presented in (2). The tire friction model is the Burckhardt [4] model that describes both longitudinal and lateral tire-forces. The vehicle model was compared with a high-performance vehicle simulator applied in the industry. Tested with some typical steering and braking excitations the measured signals showed good fit in wide range of operation.

For the controller the following signals are available as measurements: lateral $a_{y}$ and longitudinal $a_{x}$ accelerations; yaw rate $r$; steering angle $\delta$; brake pressures in the brake cylinders $p_{i j}$ and rotation equivalent wheel velocities $v_{R i j}$. From these measurements some other variables are calculated: the vehicle sideslip angle $\beta$, which is the state of the single-track model; wheel 
contact velocities $v_{w i j}$, average wheel slip angles at front and rear $\alpha_{f}$ and $\alpha_{r}$, respectively, are computed by formulas similar that is used in the high-order simulator. Observer was not used. The vertical wheel loads $F_{z i j}$ are assumed to be available by some measurements and estimation. Other methods for estimating these variables can be found in [4] and [8].

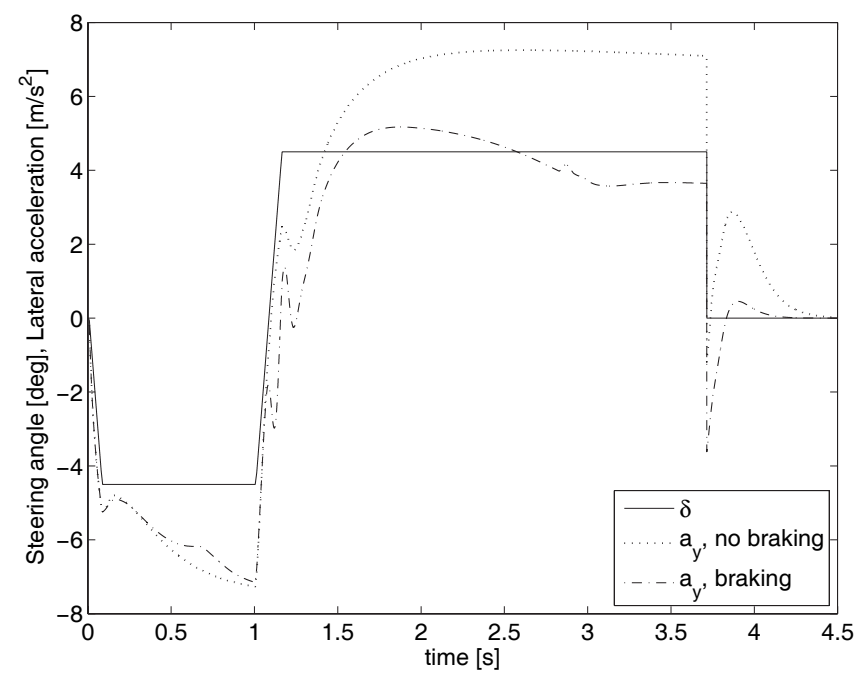

Fig. 2. Steering angle and lateral accelerations

Based on these calculated variables and the cornering stiffness function $C(\lambda, \alpha)$ the slips $x_{w i j}$ can be computed and $C(\lambda, \alpha)$ can be inverted. In case of the feedback-linearization (13) the longitudinal wheel forces $F_{x i j}$ are estimated from (2) with the help of a derivative filter $D(s)=\frac{100 s+1}{s+100}$ for the wheel velocities.

On $22 \mathrm{~m} / \mathrm{s}$ velocity a sharp left steering was applied with about 4.5 grad of steering angle for one sec. (first stage), after that a sharp right turn for a $2.5 \mathrm{~s}$ period (second stage) followed by a zero steering angle stage (3.7s-4.5s). Two simulations were applied: this steering manoeuvre with and without anti-roll braking control. There was no driving during the simulation.

The steering angle and the lateral acceleration of the two cases are shown in Fig. 2 and the vehicle slip angle, yaw rate and the velocity in Fig. 3. The lateral acceleration $a_{y}$ achieved a dangerous level if no control worked. In the second experiment the outer front and rear wheels were braked by a controller that was switched on whenever $\left|a_{y}\right|>4 \mathrm{~m} / \mathrm{s}^{2}$. It can be seen that the lateral acceleration was considerably decreased in the second stage, due to the braking.

During an overtake manoeuvre, which consists of two turns of opposite directions, the vehicle is more easily rolling over, than during a simple turn. At the first turn the body inclines towards the outer side then in the opposite turn it inclines hard to the opposite direction. The goal of the control, i.e. the avoidance of rollover can be well followed in Fig. 3 In the second stage the roll angle was much greater than in the first, when no brake
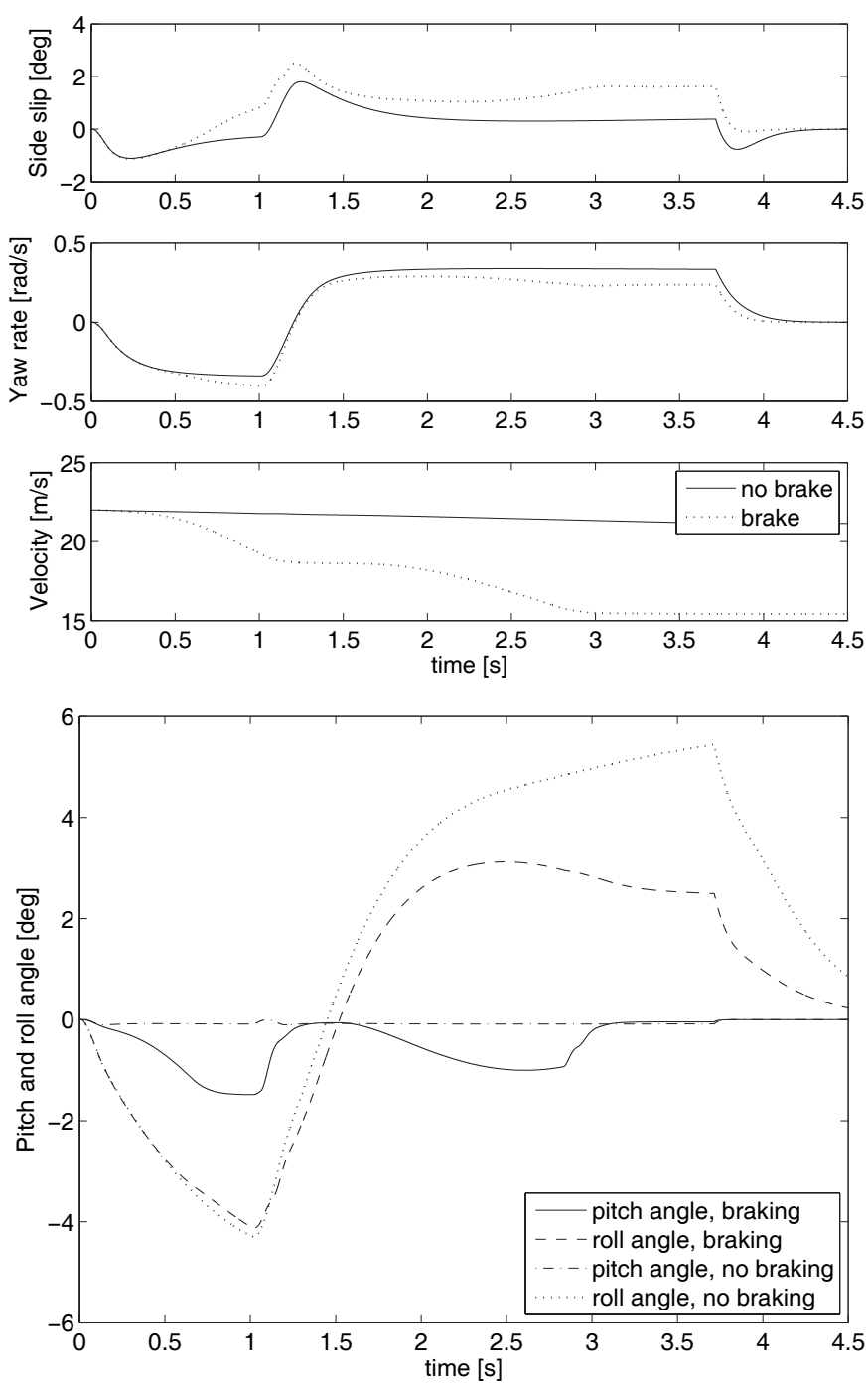

Fig. 3. Above: Side slip, yaw rate and velocity; below: Pitch and roll angles.

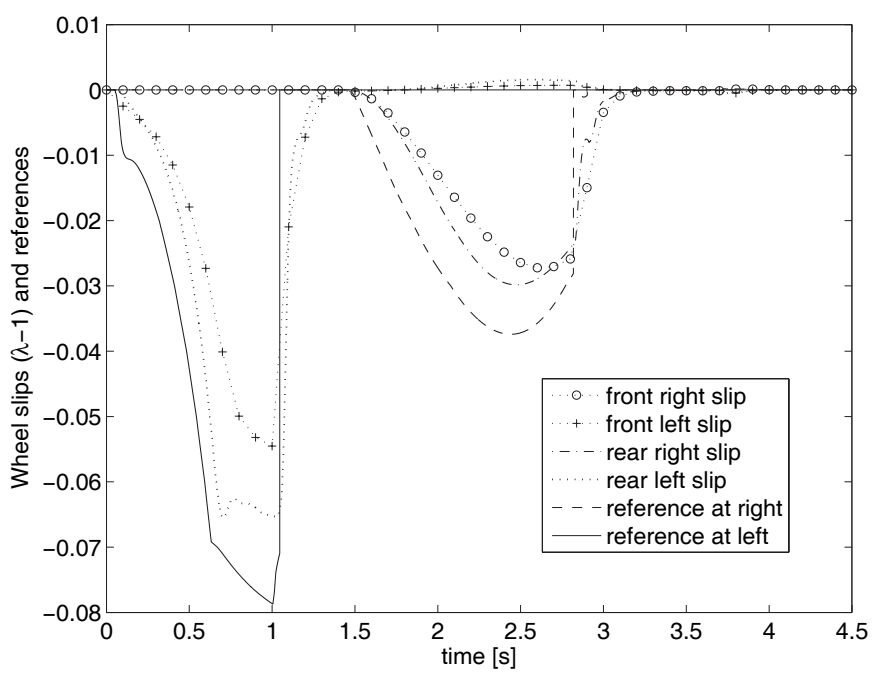

Fig. 4. Longitudinal slips, $\lambda_{i j}-1$ and its references

action was allowed (dotted line). In the second stage the roll angle became much smaller due to the braking.

It can be seen in Fig. 3 that the yaw rate slightly increased 


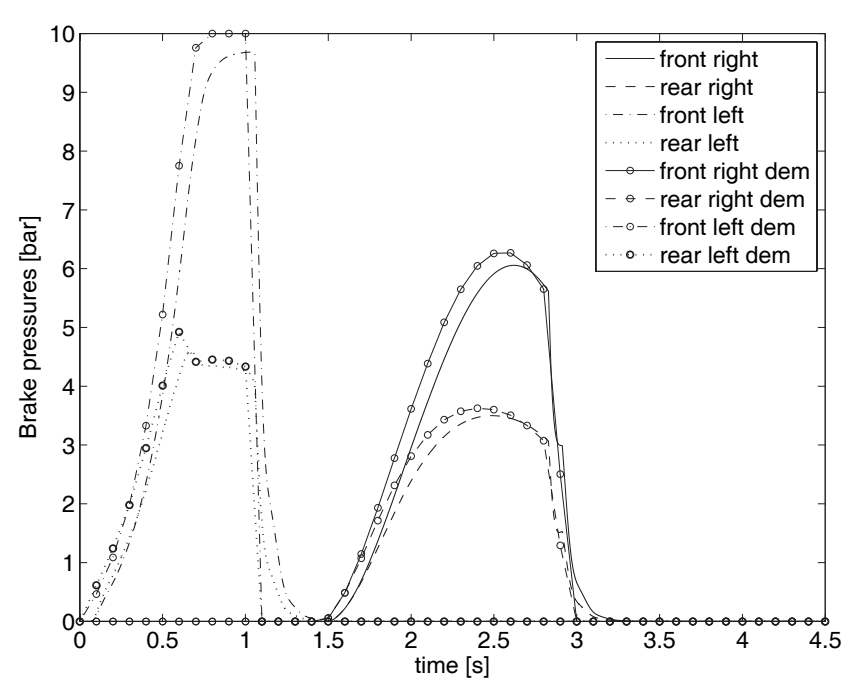

Fig. 5. Brake pressures and its references

in the first stage. The slip control could not increase the longitudinal slip of the front left wheel to the reference (see Fig. 4), because the pressure demand of the actuator saturated (Fig. 55. Due to the longitudinal forces in Fig. 6, the load shifted from the rear to the front wheels and due to the right turn it shifted from right to the left. On high velocity and large load on the front left wheel the 10 bar brake pressure cannot block the wheel. Fig. 7 shows that the lateral force of the front left wheel slightly increased, instead of decreasing, meanwhile that of the rear left wheel increased as expected. As a result the rear of the vehicle slipped sideways and the yaw rate slightly increased. However, due to the robustness of the controller the saturation was tolerated and the lateral acceleration decreased.

In the second stage $(1 \mathrm{~s}-3.7 \mathrm{~s})$ the right wheels are braked. The velocity decreased to $18 \mathrm{~m} / \mathrm{s}$ and due to this the lateral forces (Fig. 7) are a bit smaller before the second starting of the controller at time $t=1.5 \mathrm{~s}$ and less brake forces (Fig. 5) are enough to reduce the lateral acceleration.

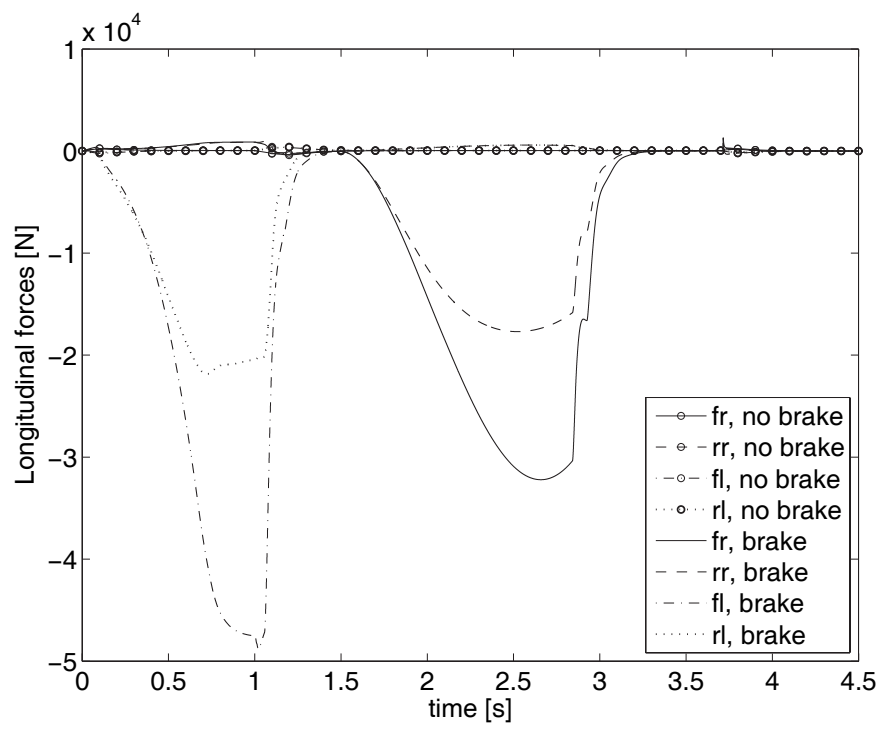

Fig. 6. Longitudinal tire-road contact forces
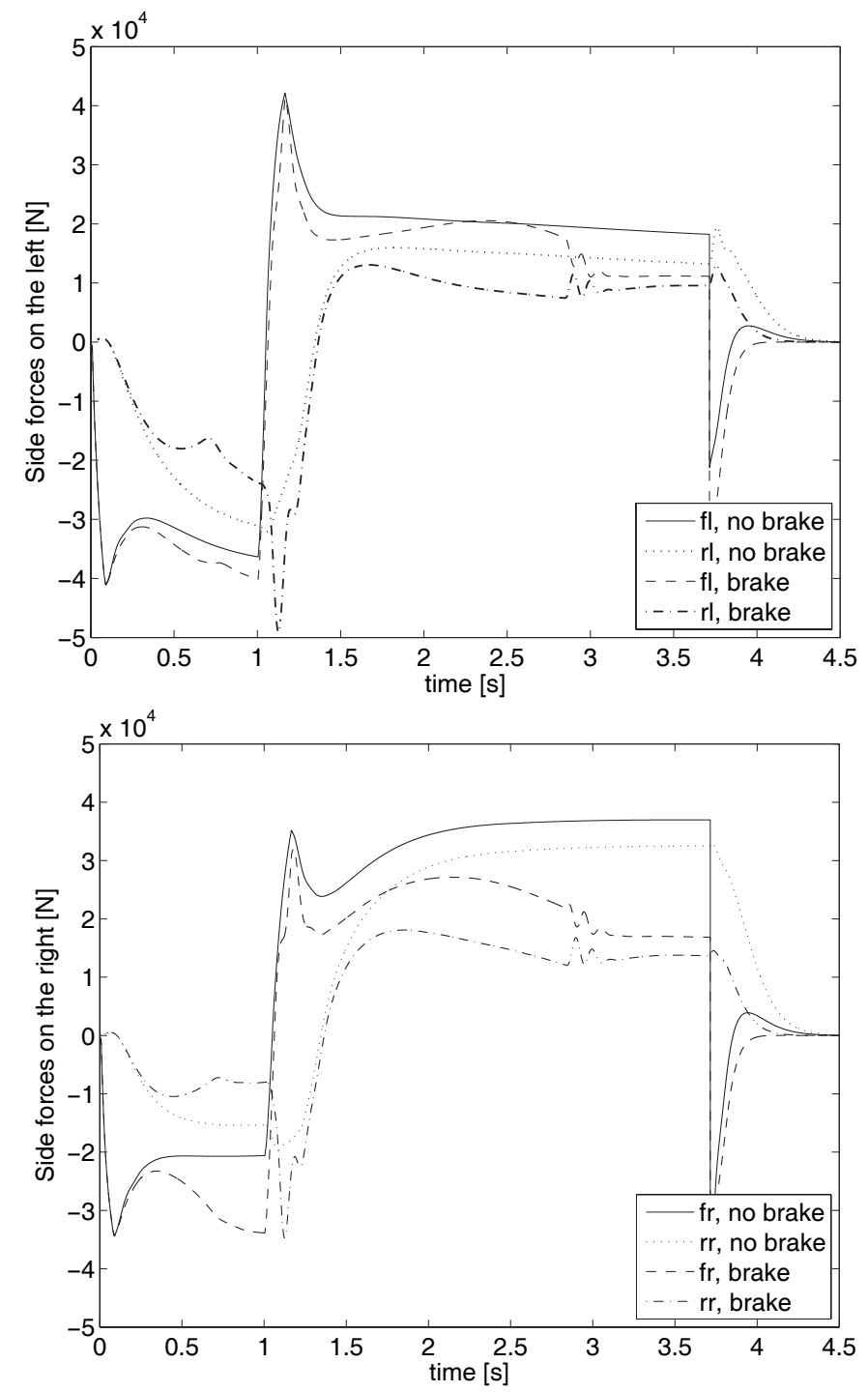

Fig. 7. Lateral tire-road contact forces on the left and the right sides

\section{Conclusion}

A three level cascade control scheme is presented for vehicle stability enhancement. In order to avoid rolling over the controller reduces the lateral acceleration as soon as it exceeds a certain limit. The switching and the disturbance feed-forward problem is performed using a simple dead-zone nonlinearity applied on the lateral acceleration measurement. The vehicle model is decomposed into yaw dynamics, wheel dynamics and brake actuator dynamics. The nonlinear wheel model is linearized. Robust $\mathcal{H}_{\infty}$ controllers are designed for the three linear systems. The algorithm is shown to work well in a high velocity overtaking manoeuvre.

\section{References}

1 Abe M, A study on effects of roll moment distribution control in active suspension on improvement of limit performance of vehicle handling, Int. Journal of Vehicle Design 15 (1994), 326-336.

2 Ackermann J, Odenthal D, Damping of vehicle roll dynamics by gain scheduled active steering, Proc. European Control Conference (1999). 
3 Ackermann J, Odenthal D, Bunte T, Advantages of active steering for vehicle dynamics control, Proc. Int. Symposium on Automotive Technology and Automation, Vienna (1999).

4 Burckhardt M, Radschlupf-Regelsysteme, Vogel Fachbuchgruppe: Fahrwerktechnik. Vogel Buchverlag, Würzburg (1993).

5 Chen B, Peng H, Differential-braking-based rollover prevention for sport utility vehicles with human-in-the-loop evaluations, Vehicle System Dynamics 36, No. 4-5 (2001), 359-389.

6 Frank P, Palkovics L, Gianone P, Using wheel speed and wheel slip information for controlling vehicle chassis systems, Proc. of the 5th International Symposium on Advanced Vehicle Control, Ann Arbor (2000).

7 Hiemer M, Model based detection and reconstruction of road traffic accidents, Universität Karlsruhe, Fak. f. Elektrotechnik und Informationstechnik. Dissertation (2004).

8 Kiencke U, Nielsen L, Automotive control systems. For engine, driveline and vehicle, Springer (2000).

9 Lin RC, Cebon $Đ$, Cole DJ, Optimal roll control of a single-unit lorry, Proc. IMechE, Journal of Automobile Engineering 210 (1996), 44-55.

10 Palkovics L, Semsey A, Gerum E, Roll-over prevention system for commercial vehicles - Additional sensorless function of the electronic brake system, Vehicle System Dynamics 32 (1999), 285-297.

11 Rödönyi G, A dynamic model of a heavy truck, Computer and Automation Research Institute Hungarian Academy of Sciences, Systems and Control Laboratory, 2007.

12 Sampson DJM, Cebon D, Active roll control of single unit heavy road vehicles, Vehicle System Dynamics 40 (2003), 229-270. 